\title{
A CHECKLIST OF THE VASCULAR FLORA OF THE MARY K. OXLEY NATURE CENTER, TULSA COUNTY, OKLAHOMA
}

\author{
Amy K. Buthod \\ Oklahoma Biological Survey \\ Oklahoma Natural Heritage Inventory \\ Robert Bebb Herbarium \\ University of Oklahoma \\ Norman, OK 73019-0575 \\ (405) 325-4034
}

Email: amybuthod@ou.edu

Keywords: flora, exotics, inventory

\begin{abstract}
This paper reports the results of an inventory of the vascular flora of the Mary K. Oxley Nature Center in Tulsa, Oklahoma. A total of 342 taxa from 75 families and 237 genera were collected from four main vegetation types. The families Asteraceae and Poaceae were the largest, with 49 and 42 taxa, respectively. Fifty-eight exotic taxa were found, representing 17\% of the total flora. Twelve taxa tracked by the Oklahoma Natural Heritage Inventory were present.

\section{INTRODUCTION}

The objective of this study was to inventory the vascular plants of the Mary $\mathrm{K}$. Oxley Nature Center (ONC) and to prepare a list and voucher specimens for Oxley personnel to use in education and outreach. Located within the 1,165.0 ha (2878 ac) Mohawk Park in northwestern Tulsa County (ONC headquarters located at 36²'25.02'N 9554'11.95'W; grounds include T20N R13E, parts of sections 10, $11,14-16)$, ONC is operated by the City of Tulsa. The 325.4 ha $(804 \mathrm{ac})$ Center includes over $14.5 \mathrm{~km}$ (9.0099 mi) of trails traversing forest, grassland, and wetland habitats.

ONC is located in the Claremore Cuesta Plains geomorphic province (Curtis et al. 2008). Quaternary sediments overlie Pennsylvanian bedrock (Johnson 2008). Soils belong to the Osage-Wynona association and are described as "deep and nearly level, poorly to somewhat poorly drained and loamy or clayey over loamy or

clayey sediment (USDA Soil Conservation Service 1977). Climate is Subtropical Humid, and summers are humid and warm with a mean July temperature of $27.5^{\circ} \mathrm{C}$ $\left(81.5^{\circ} \mathrm{F}\right)$. Winters are mild and short with a mean January temperature of $1.5^{\circ} \mathrm{C}$ $\left(34.7^{\circ} \mathrm{F}\right)$ (Trewartha 1968). Mean annual precipitation is $106.5 \mathrm{~cm}$ (41.929 in), with most occurring in the spring and fall (Oklahoma Climatological Survey 2013). Elevation ranges from 170.7 to $312.1 \mathrm{~m}$ $(560.04 \mathrm{ft}$ to $1024 \mathrm{ft})$. Potential natural vegetation for the area is tallgrass prairie and post oak-blackjack oak forest (Duck and Fletcher 1943).

\section{METHODS}

The ONC trail system was walked to determine the major, generalized vegetation types that were present, and 11 collection areas (corresponding to the named trails) were established to represent these types. Sites were visited monthly from March
\end{abstract}


through September of 2010, with additional visits made in June and September of 2011. Two specimens of each taxa encountered were collected. Exotic taxa were only collected from naturalized populations. Specimens were processed following standard procedures and deposited at the Robert Bebb Herbarium at the University of Oklahoma (OKL) and at ONC.

Manuals used for specimen identification included Steyermark (1963), Waterfall (1973), Flora of North America Editorial Committee (1993+), and Yatskievych (1999, 2006). The collections of the Robert Bebb Herbarium were consulted to confirm identifications. Origin (either native or exotic to North America), duration (annual, perennial, biennial), and growth habit (tree, shrub, subshrub, woody vine, forb, graminoid) were determined using the PLANTS Database (USDA-NRCS 2007) and Taylor and Taylor (1991). Nomenclature primarily follows the PLANTS Database. The Angiosperm Phylogeny Website, version 12 (Stevens 2001+), and the Flora of North America
North of Mexico (Flora of North America Editorial Committee 1993+) were also consulted to determine where the nomenclature/taxonomy differed from USDA-NRCS; these differences are noted in parentheses in the appendix.

\section{RESULTS AND DISCUSSION}

A total of 342 vascular plant taxa (including 23 infraspecific taxa) in 75 families and 237 genera were collected at ONC (Appendix). All were angiosperms, with 85 Liliopsida taxa and 257 Magnoliopsida (Table 1). Sixty-one taxa were trees, shrubs, subshrubs, or woody vines. The Asteraceae, with 49 taxa, and the Poaceae, with 42 taxa, were the largest families. The genus with the most taxa was Carex, with 12 taxa. There were 236 perennials, 103 annuals, and three biennials. ONC Director Eddie Reese (pers. comm.). has reported two additional taxa (Botrychium virginianum in the Ophioglossaceae and Juniperus virginiana in the Cupressaceae), but these were not collected by the author.

Table 1 Summary of floristic collections from the Mary K. Oxley Nature Center, Tulsa County, Oklahoma

\begin{tabular}{|l|l|l|l|}
\hline Taxonomic Group & Taxa & Native & Non-native \\
\hline Pteridophyta & 0 & 0 & 0 \\
\hline Coniferophyta & 0 & 0 & 0 \\
\hline Magnoliophyta & 342 & 284 & 58 \\
\hline Magnoliopsida & 257 & 215 & 42 \\
\hline Liliopsida & 85 & 69 & 16 \\
\hline Total & 342 & 284 & 58 \\
\hline
\end{tabular}


Fifty-eight exotic taxa were found, representing $17.0 \%$ of the total flora. This number is high when compared to surveys from other Oklahoma sites (Table 2), but is not surprising, given that $\mathrm{ONC}$ is in the Tulsa metropolitan area and receives many visitors. The Poaceae and Fabaceae families had the greatest number of exotic taxa, with 14 and 8 taxa, respectively. Twelve taxa tracked by the Oklahoma Natural Heritage Inventory (2013) were present (Table 3).

Table 2 Comparison of the ONC site with other northeastern Oklahoma sites $\mathrm{NA}=$ information not available

\begin{tabular}{|c|c|c|c|c|}
\hline Study site & Reference & $\begin{array}{l}\text { Size of site } \\
\text { (ha) }\end{array}$ & $\begin{array}{l}\text { Number of } \\
\text { taxa found }\end{array}$ & $\begin{array}{l}\text { Percentage } \\
\text { of exotic } \\
\text { taxa }\end{array}$ \\
\hline $\begin{array}{l}\text { Mary K. Oxley Nature } \\
\text { Center, Tulsa County }\end{array}$ & This paper & $1,165.0$ & 342 & $17.0 \%$ \\
\hline $\begin{array}{l}\text { J.T. Nickel Family } \\
\text { Nature and Wildlife } \\
\text { Preserve, Cherokee } \\
\text { County }\end{array}$ & $\begin{array}{l}\text { Hoagland and Buthod } \\
2008\end{array}$ & $6,070.0$ & 597 & $12.1 \%$ \\
\hline $\begin{array}{l}\text { Camp Cherokee Boy } \\
\text { Scout Camp, Ottawa } \\
\text { County }\end{array}$ & $\begin{array}{l}\text { Hoagland and Buthod } \\
2008\end{array}$ & $\mathrm{NA}$ & 318 & $10.4 \%$ \\
\hline $\begin{array}{l}\text { Oklahoma Centennial } \\
\text { Botanic Garden, } \\
\text { Osage County }\end{array}$ & $\begin{array}{l}\text { Hoagland and Buthod } \\
2007\end{array}$ & NA & 293 & $15.0 \%$ \\
\hline $\begin{array}{l}\text { Will Rogers Boy Scout } \\
\text { Camp, Pawnee County }\end{array}$ & $\begin{array}{l}\text { Hoagland and Buthod } \\
2005\end{array}$ & 64.7 & 338 & $8.0 \%$ \\
\hline $\begin{array}{l}\text { Keystone Wildlife } \\
\text { Management Area, } \\
\text { Creek, Pawnee and } \\
\text { Osage Counties }\end{array}$ & $\begin{array}{l}\text { Hoagland and Buthod } \\
2003\end{array}$ & $6,475.0+$ & 380 & $15.0 \%$ \\
\hline $\begin{array}{l}\text { Chouteau Wildlife } \\
\text { Management Area, } \\
\text { Wagoner County }\end{array}$ & $\begin{array}{l}\text { Hoagland and Johnson } \\
2004\end{array}$ & 402.0 & 181 & $11.6 \%$ \\
\hline $\begin{array}{l}\text { Oologah Wildlife } \\
\text { Management Area, } \\
\text { Nowata County }\end{array}$ & $\begin{array}{l}\text { Hoagland and Wallick } \\
2003\end{array}$ & $5,226.0$ & 470 & $9.0 \%$ \\
\hline $\begin{array}{l}\text { Tallgrass Prairie } \\
\text { Preserve, Osage } \\
\text { County }\end{array}$ & Palmer 2007 & $15,410.0$ & 1,612 & $12.1 \%$ \\
\hline
\end{tabular}


Table 3 Vascular plant taxa tracked by the Oklahoma Natural Heritage Inventory (2013). Conservation ranks are based on a scale from one to five, with a one being critically imperiled. A $\mathrm{G}$ rank is the rank for the taxa at a global level, while an $\mathrm{S}$ rank is one for the taxa at a subnational (or state) level. T ranks are rankings for infraspecific taxa (NatureServe 2013).

\begin{tabular}{|l|l|l|l|}
\hline Taxa & Family & S Rank & G Rank \\
\hline Lactuca tatarica var. pulchella & Asteraceae & S1 & G5T5 \\
\hline Iodanthus pinnatifidus & Brassicaceae & S2 & G5 \\
\hline Desmodium illinoense & Fabaceae & S3 & G5 \\
\hline Desmodium pauciflorum & Fabaceae & S1 & G5 \\
\hline Calycocarpum lyonii & Menispermaceae & S2 & G5 \\
\hline Mublenbergia bushii & Poaceae & S1 & G5 \\
\hline Rumex verticillatus & Polygonaceae & S3 & G5 \\
\hline Thalictrum revolutum & Ranunculaceae & S2 & G5 \\
\hline Potentilla rivalis & Rosaceae & S1 & G5 \\
\hline Dasistoma macrophylla & Scrophulariaceae & S3 & G4 \\
\hline Smilax lasioneura & Smilacaeae & S2 & G5 \\
\hline Urtica chamaedryoides & Urticaceae & S3 & G4G5 \\
\hline
\end{tabular}

Collection sites were located within four vegetation types:

\section{Mesic Forest [MF] ) (Figure 1)}

This type included the Sierra Club Trail and the North Woods areas. Common taxa included Campanulastrum americanum, Cardamine concatenata, Cercis canadensis, Cornus drummondii, Diarrhena obovata, Diospyros virginiana, Erythronium albidum, Eutrochium purpureum, Lindera benzoin, Osmorbiza longistylis, Polygonatum biflorum, and Thalictrum revolutum. Dominant taxa included Carya cordiformis, Quercus mueblenbergii, and $Q$. shumardii. The most tracked taxa were found in this type.

\section{Bottomland Forest [BF] (Figure 2)}

This type included the Bird Creek, Green Dragon, and Red Fox trails. Common taxa included Acer negundo, A. saccharinum, Celtis laevigata, Cinna arundinacea, Elephantopus carolinianus, Quercus palustris, Parthenocissus quinquefolia, and Ulmus americana. Dominant taxa included Chasmanthium latifolium, Fraxinus pennsylvanica, and Ilex decidua.

\section{Disturbed Herbaceous Vegetation [DHV] (Figure 3)}

This type included the Meadowlark Prairie trail and various disturbed areas throughout ONC. Grassland elements, including Andropogon gerardii, Panicum virgatum, and Sorghastrum nutans, were present, as were more "weedy" taxa such as Amphiachyris dracunculoides, Carduus nutans, and Ambrosia artemisiifolia. Desmanthus illinoenesis, Iva annua, and Rhus glabra were dominant taxa. The greatest number of exotic taxa were found in this type.

\section{Wetland and Aquatic Vegetation [WAV] (Figure 4)}

This type included the Bird Creek, Blackbird Marsh, Coal Creek, Flowline, and Nelson's Oxbow trails, as well as the margins of Lake Sherry and Lake Yahola. Common taxa included Carex lupulina, Eleocharis obtusa, Juncus effusus, Justicia americana, Limnosciadium pinnatum, Ludwigia peploides, and Sagittaria graminea. Nelumbo lutea and Typha angustifolia were dominant taxa in this type. 


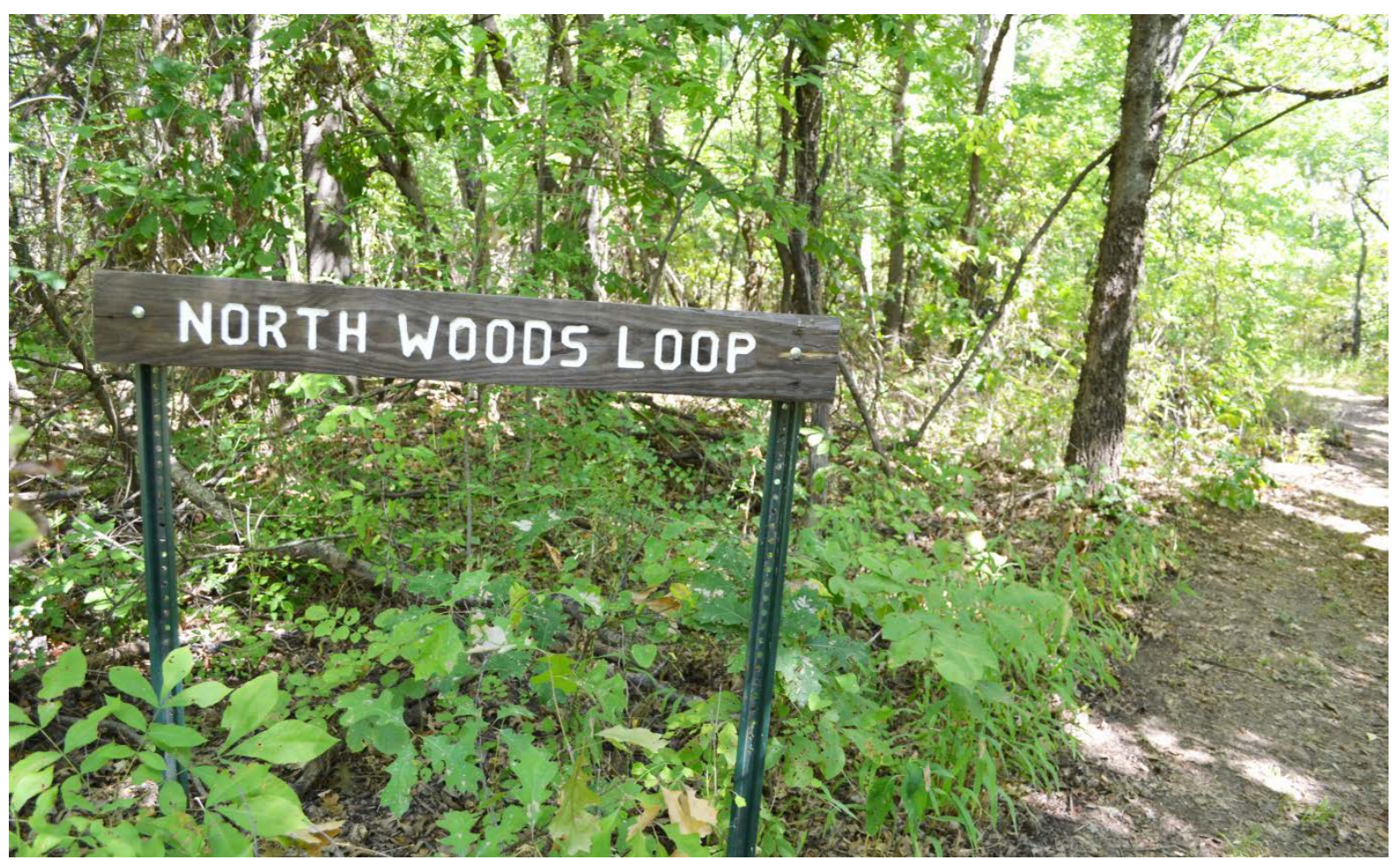

Figure 1 Mesic forest in North Woods Loop at the Mary K. Oxley Nature Center, Tulsa County, Oklahoma

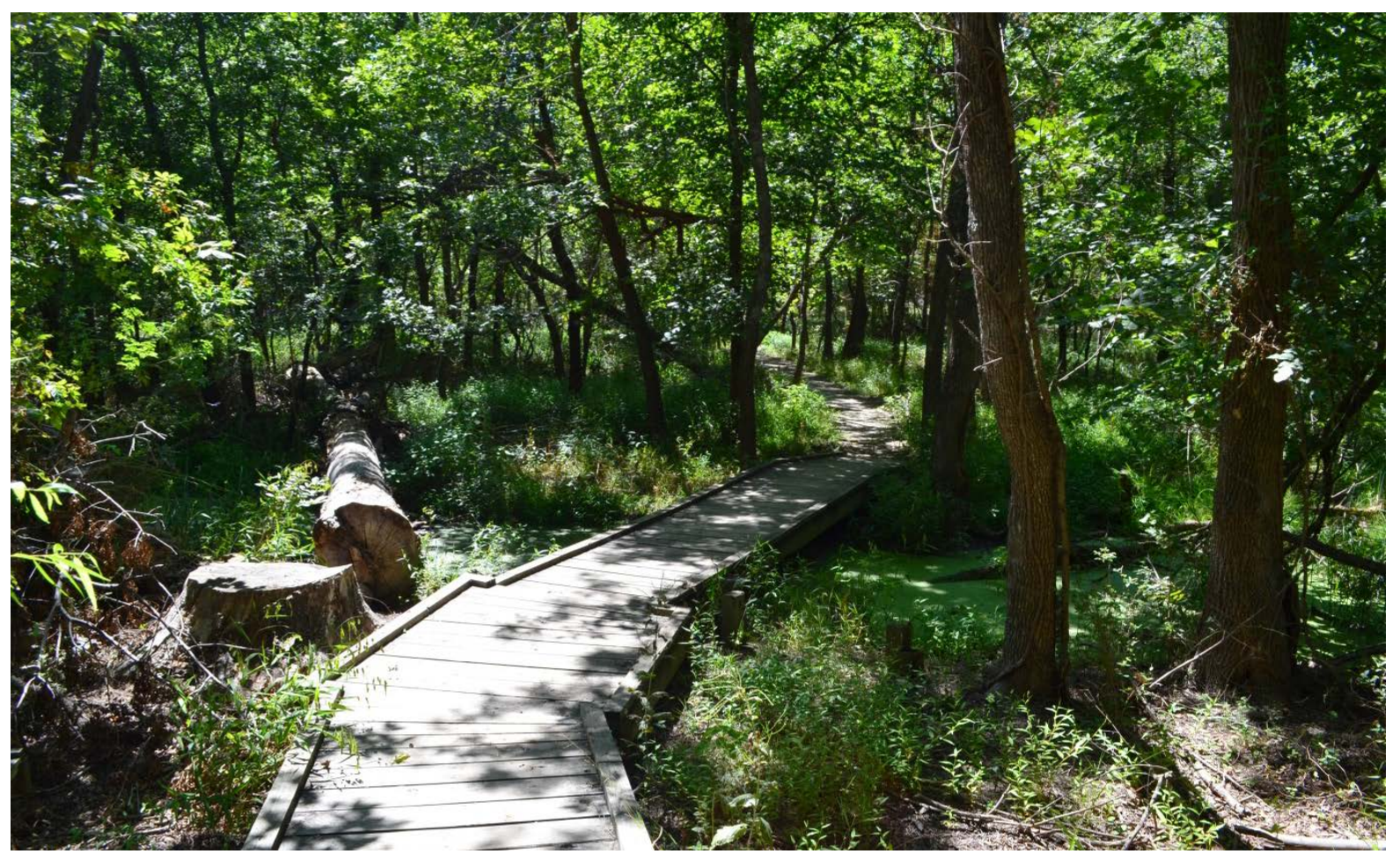

Figure 2 Bottomland forest habitat at the Mary K. Oxley Nature Center 


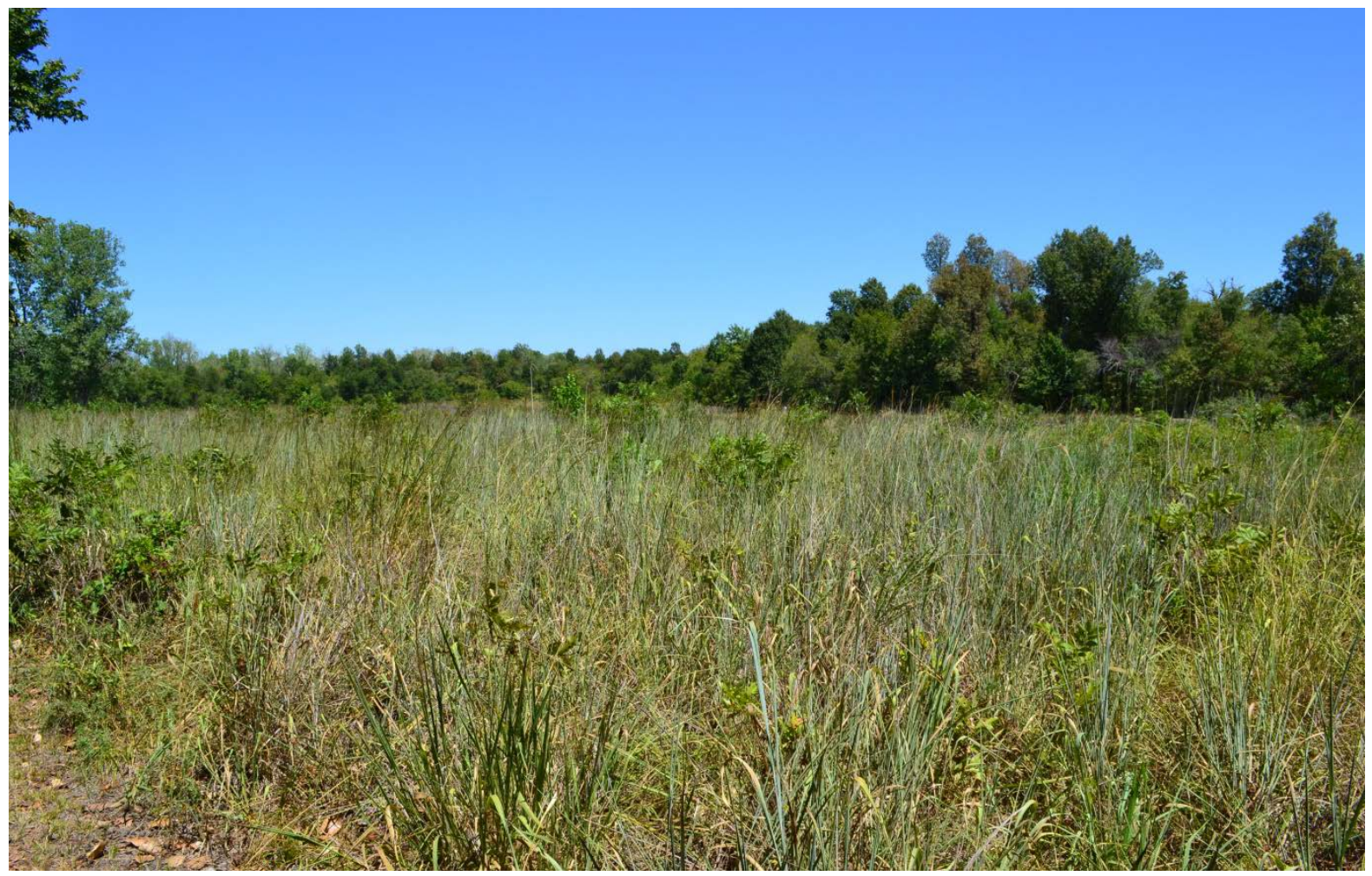

Figure 3 Disturbed herbaceous vegetation in Meadowlark Prairie at the Mary K. Oxley Nature Center

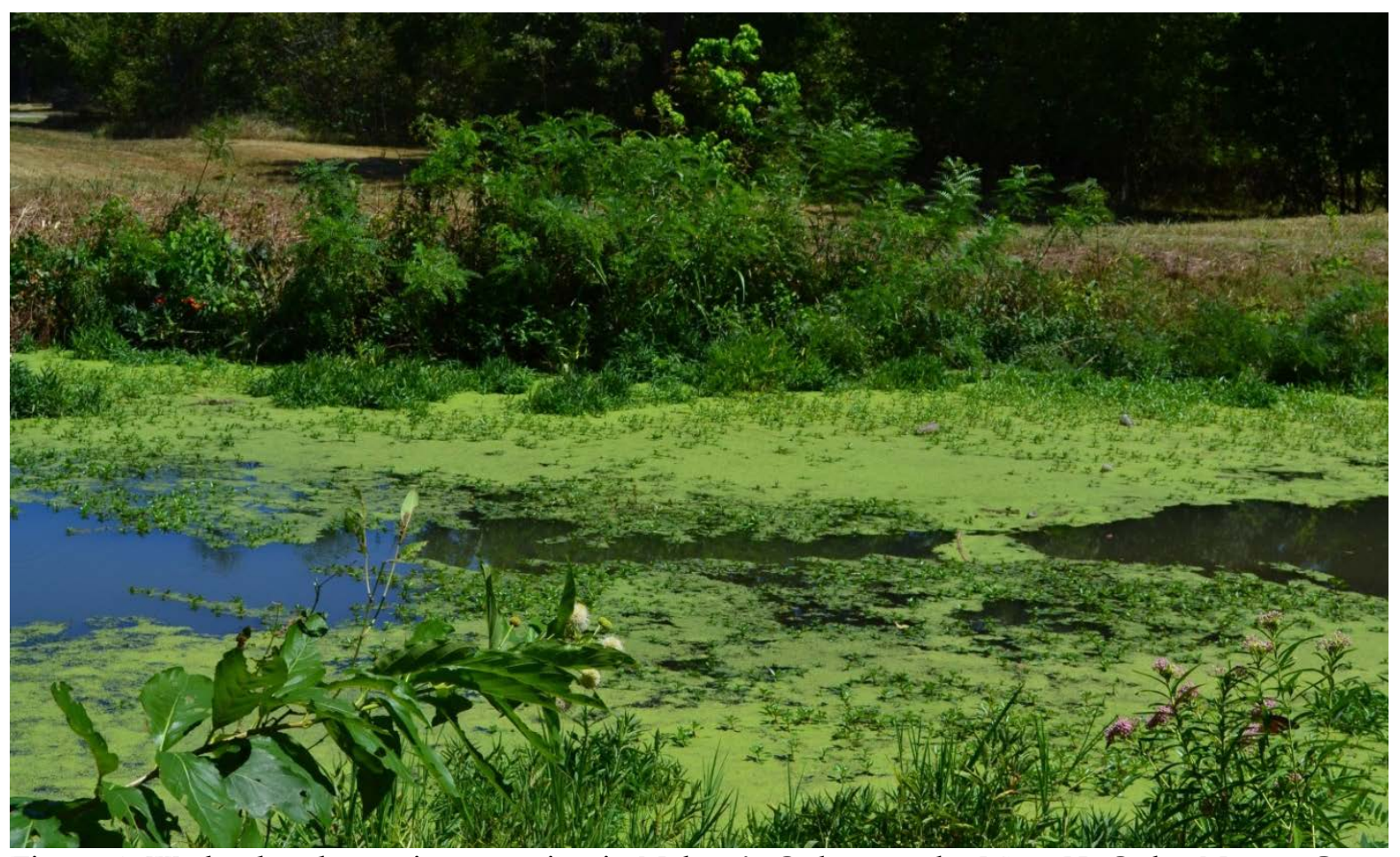

Figure 4 Wetland and aquatic vegetation in Nelson's Oxbow at the Mary K. Oxley Nature Center 


\section{ACKNOWLEDGMENTS}

The author would like to thank the Mary K. Oxley staff for allowing her access to the Center. Bruce Hoagland and Todd Fagin (Oklahoma Biological Survey/Oklahoma Natural Heritage Inventory/University of Oklahoma Department of Geography and Environmental Sustainability) provided technical assistance. This work was funded by the Oklahoma Biological Survey.

\section{LITERATURE CITED}

Curtis, N.M., W.E. Ham, and K.S. Johnson. 2008. Geomorphic provinces of Oklahoma. In: Johnson, K.S. and K.V. Luza (editors). Earth Sciences and Mineral Resources of Oklahoma. Norman (OK): Oklahoma Geological Survey.

Duck, L.G. and J.B. Fletcher. 1943. A game type map of Oklahoma. In: Duck, L.G. and J.B. Fletcher (editors). A Survey of the Game and Furbearing Animals of Oklahoma. Oklahoma City (OK): Oklahoma Department of Wildlife Conservation.

Flora of North America Editorial Committee (editors). 1993+. Flora of North America North of Mexico. 16+ vols. New York and Oxford.

Hoagland, B.W. and A.K. Buthod. 2008. The vascular flora of the J.T. Nickel Family Nature and Wildlife Preserve, Cherokee County, Oklahoma. Castanea 73(1):16-28.

Hoagland, B.W. and A.K. Buthod. 2008. The vascular flora of an Ozark plateau site, Ottawa County, Oklahoma. Southeastern Naturalist 7(4):581-594.

Hoagland, B.W. and A.K. Buthod. 2007. The vascular flora of the Oklahoma Centennial Botanic Garden site, Osage County, Oklahoma. Oklahoma Native Plant Record 7(1):54-66.

Hoagland, B.W. and A.K. Buthod. 2005. Vascular flora of a site along the Arkansas River, Pawnee County,
Oklahoma. Oklahoma Native Plant Record 5(1):61-72.

Hoagland, B.W. and A.K. Buthod. 2003. Vascular flora of the Keystone Wildlife Management Area, Creek, Pawnee, and Osage Counties, Oklahoma. Oklahoma Native Plant Record 3(1):23-37.

Hoagland, B.W. and F. Johnson. 2004. Vascular flora of the Chouteau Wildlife Management Area, Wagoner County, Oklahoma. Oklahoma Native Plant Record 4(1):30-39.

Hoagland, B.W. and K. Wallick. 2003. Vascular flora of Oologah Wildlife Management Area in Nowata County, OK. Proceedings of the Oklahoma Academy of Science 83:47-62.

Johnson, K.S. 2008. Geologic map of Oklahoma. In: Johnson, K.S. and K.V. Luza (editors). Earth Sciences and Mineral Resources of Oklahoma. Norman (OK): Oklahoma Geological Survey.

Natureserve. 2013. NatureServe Explorer: An Online Encyclopedia of Life, version 7.0. Arlington (VA): NatureServe. (http://ww.natureserve.org/explorer accessed 1 July 2013).

Oklahoma Climatological Survey. 2013. Oklahoma Climatological Data. Norman (OK): University of Oklahoma. (www.ocs.ou.edu accessed 28 June 2013).

Oklahoma Natural Heritage Inventory. 2013. Oklahoma Natural Heritage Inventory Working List of Rare Oklahoma Plants. Norman (OK): University of Oklahoma. (www.biosurvey.ou.edu/publicat.html accessed 28 June 2013).

Palmer, M. W. 2007. The vascular flora of the Tallgrass Prairie Preserve, Osage County, Oklahoma. Castanea 72(4):235246.

Steyermark, J.A. 1963. Flora of Missouri. Ames (IA): Iowa State University Press. Stevens, P.F. 2001+. Angiosperm Phylogeny Website, version 12. St. Louis 
(MO): Missouri Botanical Garden. (http://www.mobot.org/MOBOT/rese arch/APweb/ accessed 1 July 2013).

Taylor, R.J. and C.E.S. Taylor. 1991. An Annotated List of the Ferns, Fern Allies, Gymnosperms and Flowering Plants of Oklahoma. Durant (OK): Southeastern State University.

Trewartha, G.T. 1968. An Introduction to Climate. New York: McGraw-Hill.

USDA-NRCS. 2007. The PLANTS

Database. Baton Rouge (LA): National Plant Data Center. (http://plants.usda.gov accessed 1 July 2013).
USDA Soil Conservation Service. 1977. Soil Survey of Tulsa County, Oklahoma. Washington (DC): United States Department of Agriculture, Soil Conservation Service.

Waterfall, U.T. 1973. Keys to the Flora of Oklahoma. Stillwater (OK): selfpublished.

Yatskievych, G. 1999. Steyermark's Flora of Missouri, Volume 1. St. Louis (MO): Missouri Botanical Garden Press.

Yatskievych, G. 2006. Steyermark's Flora of Missouri, Volume 2. St. Louis (MO): Missouri Botanical Garden Press 


\section{APPENDIX \\ List of Vascular Plant Taxa from the Mary K. Oxley Nature Center, Tulsa County, OK}

The first entry is duration ( $A=$ annual, $B=$ biennial, $P=$ =perennial), followed by growth habit $(T=$ tree, $\mathrm{S}=$ shrub, $\mathrm{SS}=$ subshrub, $\mathrm{V}=$ woody vine, $\mathrm{F}=$ forb, $\mathrm{G}=$ graminoid), vegetation type (MF=mesic forest, $\mathrm{BF}=$ bottomland forest, $\mathrm{DHV}=$ disturbed herbaceous vegetation, $\mathrm{WAV}=$ wetland and aquatic vegetation) and collection number. If taxonomy differs from USDA-NRCS in the Flora of North America (FNA) or the Angiosperm Phylogeny Website (APW), it is noted in parentheses ( ). Exotic taxa are denoted with an asterisk (*). Taxa tracked by the Oklahoma Natural Heritage Inventory are denoted with a dagger $(\dagger)$. Voucher specimens are deposited at the Robert Bebb Herbarium of the University of Oklahoma $(\mathrm{OKL})$ and at Oxley Nature Center (ONC).

\section{Magnoliophyta \\ Magnoliopsida \\ Acanthaceae}

Dicliptera brachiata (Pursh) Spreng. (branched foldwing); A; F; MF, BF; 0-369

Justicia americana (L.) Vahl (American water-willow); P; F; WAV; O-120

Ruellia strepens L. (limestone wild petunia); P; F; DHV; O-106

\section{Aceraceae}

Acer negundo L. (boxelder); P; T; BF; O-015; (APW:Sapindaceae)

Acer saccharinum L. (silver maple); P; T; BF; O-230; (APW:Sapindaceae)

\section{Amaranthaceae}

Amaranthus tuberculatus (Moq.) Sauer (roughfruit amaranth); A; F; DHV; O-336

Iresine rhizomatosa Standl. (Juda's bush); P; F; MF, BF; 0-368

\section{Anacardiaceae}

Rhus glabra L. (smooth sumac); P; S; DHV; O-133

Toxicodendron radicans (L.) Kuntze (eastern poison ivy); P; V; MF, BF; O-033

\section{Apiaceae}

Ammoselinum butleri (Engelm. ex S. Watson) J.M. Coult. \& Rose (Butler's sandparsley); A; F; DHV;

\section{0-165}

Chaerophyllum tainturieri Hook. (hairyfruit chervil); A; F; MF, BF; O-019

Cryptotaenia canadensis (L.) DC. (Canadian honewort); P; F; MF; O-090

Limnosciadium pinnatum (DC.) Mathias \& Constance (tansy dogshade); A; F; WAV; O-114

Osmorhiza longistylis (Torr.) DC. (longstyle sweetroot); P; F; MF; O-044

Sanicula canadensis L. (Canadian blacksnakeroot); B; F; MF; O-078

Sanicula odorata (Raf.) K.M. Pryer \& L.R. Phillippe (clustered blacksnakeroot); P; F; MF; O-077

*Torilis arvensis (Huds.) Link (spreading hedgeparsley); A; F; DHV; 0-381

Zizia aurea (L.) W.D.J. Koch (golden zizia); P; F; MF; O-040

\section{Apocynaceae}

Apocynum cannabinum L. (Indianhemp); P; F; DHV; O-129

*Vinca minor L. (common periwinkle); P; F; DHV; O-160 


\section{Aquifoliaceae}

Ilex decidua Walter (possumhaw); P; S; MF, BF; 0-030

\section{Aristolochiaceae}

Aristolochia tomentosa Sims (woolly dutchman's pipe); P; V; MF; O-319

\section{Asclepiadaceae}

Asclepias incarnata L. (swamp milkweed); P; F; WAV; O-343; (APW:Apocynaceae)

Asclepias syriaca L. (common milkweed); P; F; DHV; O-094; (APW:Apocynaceae)

Cynanchum laeve (Michx.) Pers. (honeyvine); P; F; DHV; O-344; (APW:Apocynaceae)

Matelea gonocarpos (Walter) Shinners (angularfruit milkvine); P; F; MF, BF; O-110;

(APW:Apocynaceae)

\section{Asteraceae}

Ageratina altissima (L.) King \& H. Rob. var. altissima (white snakeroot); P; F; MF; O-410

Ambrosia artemisiifolia L. (annual ragweed); A; F; DHV; 0-351

Ambrosia trifida L. (great ragweed); A; F; DHV; O-341

Amphiachyris dracunculoides (DC.) Nutt. (prairie broomweed); A; F; DHV; 0-405

Bidens polylepis S.F. Blake (bearded beggarticks); A; F; WAV; O-421

Boltonia diffusa Elliott (smallhead doll's daisy); P; F; WAV; O-413

${ }^{\star}$ Carduus nutans L. (nodding plumeless thistle); B; F; DHV; O-138

Cirsium altissimum (L.) Hill (tall thistle); B; F; DHV; O-225

Conyza canadensis (L.) Cronquist (Canadian horseweed); A; F; DHV; O-245

Conyza ramosissima Cronquist (dwarf horseweed); A; F; DHV; O-292

Coreopsis tinctoria Nutt. (golden tickseed); A; F; DHV; O-097

Dracopis amplexicaulis (Vahl) Cass. (clasping coneflower); A; F; DHV; O-108;

(FNA:Rudbeckia amplexicaulis Vahl)

Echinacea purpurea (L.) Moench (eastern purple coneflower); P; F; DHV; O-251

Eclipta prostrata (L.) L. (false daisy); A; F; WAV; O-125

Elephantopus carolinianus Raeusch. (Carolina elephantsfoot); P; F; MF, BF; 0-310

Erigeron annuus (L.) Pers. (eastern daisy fleabane); A; F; DHV; O-150

Erigeron philadelphicus L. var. philadelphicus (Philadelphia fleabane); P; F; DHV; 0-067

Eupatorium serotinum Michx. (lateflowering thoroughwort); P; F; DHV; O-332

Eutrochium purpureum (L.) E.E. Lamont var. purpureum (sweetscented joe pye weed); P; F; MF;

O-268a

Gamochaeta purpurea (L.) Cabrera (spoonleaf purple everlasting); P; F; DHV; O-099

Helianthus tuberosus L. (Jerusalem artichoke); P; F; MF; O-408

Iva annua L. (annual marshelder); A; F; DHV; O-315

Krigia caespitosa (Raf.) K.L. Chambers (weedy dwarfdandelion); A; F; DHV; O-053;

(FNA:Krigia cespitosa)

Lactuca canadensis L. (Canada lettuce); A; F; DHV; O-269

Lactuca floridana (L.) Gaertn. (woodland lettuce); A; F; MF; O-313

*Lactuca serriola L. (prickly lettuce); A; F; DHV; O-353

†Lactuca tatarica (L.) C.A. Mey. var. pulchella (Pursh) Breitung (blue lettuce); P; F; DHV; O-375;

(FNA:Mulgedium pulchellum (Pursh) G. Don)

Packera glabella (Poir.) C. Jeffrey (butterweed); A; F; BF; 0-066

Packera obovata (Muhl. ex Willd.) W.A. Weber \& Á. Löve (roundleaf ragwort); P; F; MF, BF; 0-008 
Pluchea camphorata (L.) DC. (camphor pluchea); P; F; WAV; O-309

Pyrrhopappus carolinianus (Walter) DC. (Carolina desert-chicory); A; F; DHV; 0-113

Ratibida pinnata (Vent.) Barnhart (pinnate prairie coneflower); P; F; DHV; O-385

Rudbeckia hirta L. var. pulcherrima Farw. (blackeyed Susan); P; F; DHV; 0-121

Rudbeckia triloba L. var. triloba (browneyed Susan); P; F; MF; 0-415

Silphium asteriscus L. var. asteriscus (starry rosinweed); P; F; DHV; O-271

Silphium perfoliatum L. var. perfoliatum (cup plant); P; F; MF; O-235

Smallanthus uvedalius (L.) Mackenzie ex Small (hairy leafcup); P; F; MF; 0-350;

(FNA:Smallanthus uvedalia (L.) Mack. ex Small)

Solidago gigantea Aiton (giant goldenrod); P; F; DHV; 0-373

Solidago speciosa Nutt. var. rigidiuscula Torr. \& A. Gray (showy goldenrod); P; F; DHV; O-293

Solidago ulmifolia Muhl. ex Willd. var. ulmifolia (elmleaf goldenrod); P; F; MF; 0-306

*Sonchus asper (L.) Hill (spiny sowthistle); A; F; DHV; O-147

Symphyotrichum cordifolium (L.) G.L. Nesom (common blue wood aster); P; F; MF; O-419

Symphyotrichum lanceolatum (Willd.) G.L. Nesom (white panicle aster); P; F; MF; O-423

Symphyotrichum subulatum (Michx.) G.L. Nesom (eastern annual saltmarsh aster); A; F; DHV, WAV; 0-305

*Taraxacum officinale F.H. Wigg. (common dandelion); P; F; DHV; O-025

Verbesina alternifolia (L.) Britton ex Kearney (wingstem); P; F; MF; O-374

Verbesina virginica L. (white crownbeard); P; F; MF; O-327

Vernonia baldwinii Torr. (Baldwin's ironweed); P; F; DHV; O-220

Xanthium strumarium L. (rough cocklebur); A; F; WAV; 0-406

\section{Bignoniaceae}

Campsis radicans (L.) Seem. ex Bureau (trumpet creeper); P; V; MF, BF; O-236

\section{Boraginaceae}

*Buglossoides arvensis (L.) I.M. Johnston (corn gromwell); A; F; DHV; O-047

*Heliotropium indicum L. (Indian heliotrope); A; F; WAV; O-328

Myosotis verna Nutt. (spring forget-me-not); A; F; BF; 0-070

\section{Brassicaceae}

${ }^{*}$ Capsella bursa-pastoris (L.) Medik. (shepherd's purse); A; F; DHV; 0-012

Cardamine concatenata (Michx.) Sw. (cutleaf toothwort); P; F; MF; 0-009

${ }^{*}$ Cardamine hirsuta L. (hairy bittercress); A; F; BF, DHV; O-018

Cardamine pensylvanica Muhl. ex Willd. (Pennsylvania bittercress); P; F; DHV; O-188

†lodanthus pinnatifidus (Michx.) Steud. (purplerocket); P; F; MF; 0-058

Lepidium virginicum L. (Virginia pepperweed); A; F; DHV; O-172

Rorippa palustris (L.) Besser ssp. palustris (bog yellowcress); A; F; WAV; 0-418

Rorippa sessiliflora (Nutt.) Hitchc. (stalkless yellowcress); A; F; BF; O-071

\section{Campanulaceae}

Campanulastrum americanum (L.) Small (American bellflower); A; F; MF; O-233

Triodanis biflora (Ruiz \& Pav.) Greene (small Venus' looking-glass); A; F; DHV; 0-100

\section{Cannabaceae}

*Humulus japonicus Siebold \& Zucc. (Japanese hop); P; F; MF; 0-349 


\section{Caprifoliaceae}

*Lonicera japonica Thunb. (Japanese honeysuckle); P; V; MF, BF; 0-380

Sambucus nigra L. ssp. canadensis (L.) R. Bolli (common elderberry); P; S; MF; O-111;

(APW:Adoxaceae)

Symphoricarpos orbiculatus Moench (coralberry); P; S; MF; O-201

Viburnum rufidulum Raf. (rusty blackhaw); P; S; MF; O-054; (APW:Adoxaceae)

\section{Caryophyllaceae}

*Arenaria serpyllifolia L. (thymeleaf sandwort); A; F; DHV; O-098

*Cerastium glomeratum Thuill. (sticky chickweed); A; F; DHV; 0-170

Sagina decumbens (Elliott) Torr. \& Gray ssp. decumbens (trailing pearlwort); A; F; DHV; O-168

Silene stellata (L.) W.T. Aiton (widowsfrill); P; F; MF; O-112

*Stellaria media (L.) Vill. (common chickweed); A; F; DHV; O-022

\section{Celastraceae}

Celastrus scandens L. (American bittersweet); P; V; MF; 0-266

*Euonymus kiautschovicus Loes. (creeping strawberry bush); P; S; MF; 0-378

\section{Chenopodiaceae}

Chenopodium album L. (lambsquarters); A; F; DHV; O-416; (APW:Amaranthaceae)

\section{Clusiaceae}

Hypericum punctatum Lam. (spotted St. Johnswort); P; F; DHV; O-217; (APW:Hypericaceae)

\section{Convolvulaceae}

Ipomoea lacunosa L. (whitestar); A; F; DHV; O-321

Ipomoea pandurata (L.) G. Mey. (man of the earth); P; F; MF; O-264

\section{Cornaceae}

Cornus drummondii C.A. Mey. (roughleaf dogwood); P; T; MF; O-032

\section{Crassulaceae}

Penthorum sedoides L. (ditch stonecrop); P; F; WAV; O-243; (APW:Penthoraceae)

\section{Cucurbitaceae}

Melothria pendula L. (Guadeloupe cucumber); P; F; DHV; O-242

\section{Cuscutaceae}

Cuscuta gronovii Willd. ex Schult. (scaldweed); P; F; DHV; O-393; (APW:Convolvulaceae)

\section{Ebenaceae}

Diospyros virginiana L. (common persimmon); P; T; MF; O-057

\section{Euphorbiaceae}

Acalypha rhomboidea Raf. (Virginia threeseed mercury); A; F; BF; 0-399

Chamaesyce nutans (Lag.) Small (eyebane); A; F; DHV; O-314

Chamaesyce prostrata (Aiton) Small (prostrate sandmat); A; F; DHV; O-250

Croton glandulosus L. (vente conmigo); A; F; DHV; O-407 
*Euphorbia davidii Subils (David's spurge); A; F; DHV; 0-346

Euphorbia dentata Michx. (toothed spurge); A; F; MF; 0-316

\section{Fabaceae}

*Albizia julibrissin Durazz. (silktree); P; T; MF; 0-311

Amorpha fruticosa L. (desert false indigo); P; S; WAV; O-357

Amphicarpaea bracteata (L.) Fernald (American hogpeanut); A; F; MF; 0-382

Apios americana Medik. (groundnut); P; F; WAV; O-234

Cercis canadensis L. (eastern redbud); $\mathrm{P} ; \mathrm{T}$; MF; O-042

Chamaecrista fasciculata (Michx.) Greene (sleepingplant); A; F; DHV; O-240

Desmanthus illinoensis (Michx.) MacMill. ex B.L. Rob. \& Fernald (prairie bundleflower); P; F; DHV;

0-237

†Desmodium illinoense A. Gray (Illinois ticktrefoil); P; F; DHV; O-329

Desmodium paniculatum (L.) DC. (panicledleaf ticktrefoil); P; F; MF; 0-105

†Desmodium pauciflorum (Nutt.) DC. (fewflower ticktrefoil); P; F; MF; O-261

Gymnocladus dioicus (L.) K. Koch (Kentucky coffeetree); P; F; MF; O-203

*Kummerowia stipulacea (Maxim.) Makino (Korean clover); A; F; DHV; O-355

*Lespedeza cuneata (Dum. Cours.) G. Don (Chinese lespedeza); P; F; DHV; O-294

Lespedeza repens (L.) W.P.C. Barton (creeping lespedeza); P; F; MF; 0-318

*Medicago lupulina L. (black medick); A; F; DHV; O-426

*Melilotus officinalis (L.) Lam. (yellow sweetclover); A; F; DHV; 0-134

Robinia pseudoacacia L. (black locust); P; T; BF; O-387

Senna marilandica (L.) Link (Maryland senna); P; F; WAV; O-320

Sesbania herbacea (Mill.) McVaugh (bigpod sesbania); A; F; WAV; 0-325

Strophostyles helvola (L.) Elliott (amberique-bean); A; F; DHV; 0-345

*Trifolium campestre Schreb. (field clover); A; F; DHV; O-088

*Trifolium repens L. (white clover); P; F; DHV; O-080

*Vicia sativa L. (garden vetch); A; F; DHV; O-173

*Vicia villosa Roth ssp. varia (Host) Corb. (winter vetch); A; F; DHV; 0-128

\section{Fagaceae}

Quercus macrocarpa Michx. (bur oak); P; T; MF, BF; 0-300

Quercus muehlenbergii Engelm. (chinkapin oak); P; T; MF; O-267a

Quercus palustris Münchh. (pin oak); P; T; BF; O-273

Quercus rubra L. (northern red oak); P; T; MF; O-298

Quercus shumardii Buckley (Shumard's oak); P; T; MF; 0-301

\section{Fumariaceae}

Corydalis flavula (Raf.) DC. (yellow fumewort); A; F; BF; O-020; (FNA:Papaveraceae)

\section{Geraniaceae}

Geranium carolinianum L. (Carolina geranium); A; F; DHV; 0-162

${ }^{*}$ Geranium pusillum L. (small geranium); A; F; DHV; O-061

\section{Juglandaceae}

Carya cordiformis (Wangenh.) K. Koch (bitternut hickory); P; T; MF; O-063

Carya illinoinensis (Wangenh.) K. Koch (pecan); P; T; MF, BF; O-048

Juglans nigra L. (black walnut); P; T; MF; O-358 


\section{Lamiaceae}

*Lamium amplexicaule L. (henbit deadnettle); A; F; DHV; O-024

*Lamium purpureum L. (purple deadnettle); A; F; DHV; O-016

Lycopus americanus Muhl. ex W.P.C. Barton (American water horehound); P; F; WAV; O-222

Monarda fistulosa L. (wild bergamot); P; F; DHV; O-383

*Perilla frutescens (L.) Britton (beefsteakplant); A; F; MF; 0-359

Prunella vulgaris L. (common selfheal); P; F; MF; 0-118

Scutellaria lateriflora L. (blue skullcap); P; F; BF; O-409

Stachys tenuifolia Willd. (smooth hedgenettle); P; F; BF; O-249

Teucrium canadense L. (Canada germander); P; F; WAV; O-210

\section{Lauraceae}

Lindera benzoin (L.) Blume (northern spicebush); P; S; MF, BF; O-014

\section{Lentibulariaceae}

Utricularia gibba L. (humped bladderwort); P; F; WAV; 0-255

\section{Lythraceae}

Ammannia auriculata Willd. (eared redstem); A; F; WAV; O-254

Lythrum alatum Pursh (winged lythrum); P; F; WAV; 0-259

\section{Malvaceae}

Callirhoe papaver (Cav.) A. Gray (woodland poppymallow); P; F; MF; 0-117

Hibiscus laevis All. (halberdleaf rosemallow); P; F; WAV; O-256

Hibiscus lasiocarpos Cav. (rosemallow); P; F; WAV; 0-246

Sida spinosa L. (prickly fanpetals); A; F; DHV; O-425

\section{Menispermaceae}

†Calycocarpum lyonii (Pursh) A. Gray (cupseed); P; V; MF; 0-115

Cocculus carolinus (L.) DC. (Carolina coralbead); P; V; MF, BF; O-390

Menispermum canadense L. (common moonseed); P; V; MF, BF; O-223

\section{Molluginaceae}

Mollugo verticillata L. (green carpetweed); A; F; DHV; O-244

\section{Moraceae}

Maclura pomifera (Raf.) C.K. Schneid. (osage orange); P; T; BF; O-339

*Morus alba L. (white mulberry); P; T; MF; O-389

Morus rubra L. (red mulberry); P; T; MF, BF; O-303

\section{Nelumbonaceae}

Nelumbo lutea Willd. (American lotus); P; F; WAV; O-229

\section{Oleaceae}

Fraxinus americana L. (white ash); P; T; MF, BF; O-265

Fraxinus pennsylvanica Marshall (green ash); P; T; BF; O-051

*Ligustrum vulgare L. (European privet); P; S; MF, BF; O-267 


\section{Onagraceae}

Gaura longiflora Spach (longflower beeblossom); A; F; DHV; 0-352

Ludwigia peploides (Kunth) P.H. Raven (floating primrose-willow); P; F; WAV; O-278

Oenothera villosa Thunb. (hairy evening-primrose); P; F; DHV; O-348

\section{Oxalidaceae}

Oxalis corniculata L. (creeping woodsorrel); P; F; DHV; O-164

Oxalis dillenii Jacq. (slender yellow woodsorrel); P; F; MF; O-089

\section{Passifloraceae}

Passiflora incarnata L. (purple passionflower); P; F; DHV; O-241

Passiflora lutea L. (yellow passionflower); P; F; MF, BF; 0-107

\section{Phytolaccaceae}

Phytolacca americana L. var. americana (American pokeweed); P; F; MF; 0-131

\section{Plantaginaceae}

*Plantago lanceolata L. (narrowleaf plantain); P; F; DHV; O-296

Plantago rugelii Decne. (blackseed plantain); P; F; MF, DHV; O-127

Plantago virginica L. (Virginia plantain); A; F; DHV; O-119

\section{Polemoniaceae}

Phlox divaricata L. (wild blue phlox); P; F; MF, BF; O-065

\section{Polygonaceae}

${ }^{*}$ Polygonum aviculare L. (prostrate knotweed); A; F; DHV; O-417

Polygonum hydropiperoides Michx. (swamp smartweed); P; F; WAV; O-274; (FNA:Persicaria hydropiperoides (Michx.) Small)

Polygonum lapathifolium L. (curlytop knotweed); A; F; WAV; O-295; (FNA:Persicaria lapathifolia (L.) Gray)

Polygonum pensylvanicum L. (Pennsylvania smartweed); A; F; WAV; O-297; (FNA:Persicaria pensylvanica (L.) M. Gómez)

Polygonum punctatum Elliott (dotted smartweed); A; F; WAV; O-219; (FNA:Persicaria punctata (Elliott) Small)

Polygonum tenue Michx. (pleatleaf knotweed); A; F; DHV; O-126

Polygonum virginianum L. (jumpseed); P; F; MF; O-212; (FNA:Persicaria virginiana (L.) Gaertn.)

Rumex altissimus Alph. Wood (pale dock); P; F; WAV; O-046

${ }^{*}$ Rumex crispus L. (curly dock); P; F; DHV; O-079

†Rumex verticillatus L. (swamp dock); P; F; WAV; 0-181

\section{Portulacaceae}

Claytonia virginica L. (Virginia springbeauty); P; F; DHV; O-005; (APW:Montiaceae)

\section{Ranunculaceae}

Clematis pitcheri Torr. \& A. Gray (bluebill); P; V; BF; O-248

${ }^{*}$ Clematis terniflora DC. var. terniflora (sweet autumn virginsbower); P; F; BF; 0-403;

(FNA:Clematis terniflora DC.)

Ranunculus micranthus Nutt. (rock buttercup); P; F; DHV; O-163 
Ranunculus sceleratus L. var. sceleratus (cursed buttercup); A; F; BF; 0-069 †Thalictrum revolutum DC. (waxyleaf meadow-rue); P; F; MF; 0-109

\section{Rosaceae}

Agrimonia pubescens Wallr. (soft agrimony); P; F; MF; O-257

Crataegus viridis L. (green hawthorn); P; T; BF; O-179

Geum canadense Jacq. (white avens); P; F; MF; O-139

Geum vernum (Raf.) Torr. \& A. Gray (spring avens); P; F; BF; 0-068

†Potentilla rivalis Nutt. (brook cinquefoil); P; F; WAV; O-384

Prunus hortulana L.H. Bailey (hortulan plum); P; T; BF; O-184

Prunus mexicana S. Watson (Mexican plum); P; T; BF; 0-095

Prunus serotina Ehrh. (black cherry); P; T; BF; 0-140

*Pyrus calleryana Decne. (Callery pear); P; T; BF; 0-331

${ }^{*}$ Pyrus communis L. (common pear); P; T; MF; O-010

Rosa setigera Michx. (climbing rose); P; V; MF; O-101

Rubus argutus Link (sawtooth blackberry); P; SS; DHV; 0-182

\section{Rubiaceae}

Cephalanthus occidentalis L. (common buttonbush); P; S; WAV; 0-205

*Cruciata pedemontana (Bellardi) Ehrend. (piedmont bedstraw); A; F; DHV; 0-060

Galium aparine L. (stickywilly); A; F; DHV; O-037

Galium circaezans Michx. (licorice bedstraw); P; F; MF, BF; 0-149

Houstonia pusilla Schoepf (tiny bluet); A; F; DHV; O-004

*Sherardia arvensis L. (blue fieldmadder); A; F; DHV; O-059

\section{Rutaceae}

Zanthoxylum americanum Mill. (common pricklyash); P; S; MF; 0-299

\section{Salicaceae}

Salix nigra Marshall (black willow); P; T; WAV; 0-178

\section{Sapotaceae}

Sideroxylon lanuginosum Michx. ssp. lanuginosum (gum bully); P; T; BF; O-324

\section{Scrophulariaceae}

†Dasistoma macrophylla (Nutt.) Raf. (mullein foxglove); A; F; MF; O-258; (APW:Orobanchaceae)

Gratiola neglecta Torr. (clammy hedgehyssop); A; F; WAV; O-072; (APW:Plantaginaceae)

Lindernia dubia (L.) Pennell (yellowseed false pimpernel); A; F; DHV; O-253; (APW:Linderniaceae)

Mimulus alatus Aiton (sharpwing monkeyflower); P; F; WAV; O-238; (APW:Phyrmaceae)

*Veronica arvensis L. (corn speedwell); A; F; DHV; O-171; (APW:Plantaginaceae)

Veronica peregrina L. (neckweed); A; F; DHV; O-174; (APW:Plantaginaceae)

*Veronica polita Fri. (gray field speedwell); A; F; BF; O-003; (APW:Plantaginaceae)

\section{Solanaceae}

Physalis angulata L. (cutleaf groundcherry); A; F; DHV; O-333

Physalis longifolia Nutt. (longleaf groundcherry); P; F; DHV; O-342

Solanum americanum P. Mill. (American black nightshade); P; F; BF; 0-400

Solanum carolinense L. (Carolina horsenettle); P; F; DHV; O-103 


\section{Ulmaceae}

Celtis laevigata Willd. (sugarberry); P; T; MF, BF; O-183

Ulmus americana L. (American elm); P; T; BF; O-002

Ulmus rubra Muhl. (slippery elm); P; T; BF; O-036

\section{Urticaceae}

Boehmeria cylindrica (L.) Sw. (smallspike false nettle); P; F; MF; O-202

Laportea canadensis (L.) Weddell (Canadian woodnettle); P; F; MF; O-260

†Urtica chamaedryoides Pursh (heartleaf nettle); A; F; MF; O-006

\section{Valerianaceae}

Valerianella radiata (L.) Dufr. (beaked cornsalad); A; F; DHV; O-038; (APW:Caprifoliaceae)

\section{Verbenaceae}

Phryma leptostachya L. (American lopseed); P; F; MF; O-231; (APW:Phrymaceae)

Phyla lanceolata (Michx.) Greene (lanceleaf fogfruit); P; F; WAV; O-086

Verbena urticifolia L. (white vervain); P; F; MF; O-228

\section{Violaceae}

Viola bicolor Pursh (field pansy); A; F; DHV; O-001

Viola missouriensis Greene (Missouri violet); P; F; BF; O-169

Viola pubescens Aiton var. pubescens (downy yellow violet); P; F; MF; O-062

Viola sororia Willd. (common blue violet); P; F; MF, BF; O-017

\section{Vitaceae}

Ampelopsis cordata Michx. (heartleaf peppervine); P; V; MF, BF; O-136

Parthenocissus quinquefolia (L.) Planch. (Virginia creeper); P; V; MF, BF; O-360

Vitis cinerea (Engelm.) Engelm. ex Millard (graybark grape); P; V; MF, BF; O-082

Vitis vulpina L. (frost grape); P; V; MF; O-155

\section{Liliopsida}

Alismataceae

Alisma subcordatum Raf. (American water plantain); P; F; WAV; 0-211

Sagittaria graminea Michx. (grassy arrowhead); P; F; WAV; O-277

Sagittaria platyphylla (Engelm.) J.G. Sm. (delta arrowhead); P; F; WAV; 0-388

\section{Araceae}

Arisaema dracontium (L.) Schott (green dragon); P; F; MF; O-116

\section{Commelinaceae}

Commelina erecta L. (whitemouth dayflower); P; F; DHV; O-322

\section{Cyperaceae}

Carex brevior (Dewey) Mack. (shortbeak sedge); P; G; DHV; 0-192

Carex caroliniana Schwein. (Carolina sedge); P; G; WAV; 0-186

Carex cherokeensis Schwein. (Cherokee sedge); P; G; BF; 0-189

Carex davisii Schwein. \& Torr. (Davis' sedge); P; G; BF; O-200

Carex granularis Muhl. ex Willd. (limestone meadow sedge); P; G; DHV; O-177

Carex hyalinolepis Steud. (shoreline sedge); P; G; BF; O-195 
Carex leavenworthii Dewey (Leavenworth's sedge); P; G; DHV; 0-197

Carex lupulina Muhl. ex Willd. (hop sedge); P; G; WAV; 0-159

Carex muehlenbergii Schkuhr ex Willd. var. muehlenbergii (Muhlenberg's sedge); P; G; DHV; 0-166

Carex retroflexa Muhl. ex Willd. (reflexed sedge); P; G; DHV; O-193

Carex scoparia Schkuhr ex Willd. var. scoparia (broom sedge); P; G; WAV; 0-154

Carex tribuloides Wahlenb. var. sangamonensis Clokey (blunt broom sedge); P; G; WAV; 0-085

Cyperus acuminatus Torr. \& Hook. ex Torr. (tapertip flatsedge); P; G; WAV; O-073

Cyperus echinatus (L.) Alph. Wood (globe flatsedge); P; G; DHV; O-209

Cyperus strigosus L. (strawcolored flatsedge); P; G; DHV, WAV; O-275

Eleocharis obtusa (Willd.) Schult. (blunt spikerush); A; G; WAV; 0-213

Eleocharis palustris (L.) Roem. \& Schult. (common spikerush); P; G; WAV; 0-398

\section{Iridaceae}

Sisyrinchium angustifolium Mill. (narrowleaf blue-eyed grass); P; F; DHV; 0-052

\section{Juncaceae}

Juncus acuminatus Michx. (tapertip rush); P; G; WAV; 0-158

Juncus brachycarpus Engelm. (whiteroot rush); P; G; WAV; O-075

Juncus diffusissimus Buckl. (slimpod rush); P; G; WAV; 0-074

Juncus effusus L. (common rush); P; G; WAV; O-135

Juncus interior Wiegand (inland rush); P; G; DHV, WAV; 0-087

Juncus tenuis Willd. (poverty rush); P; G; DHV; O-152

\section{Lemnaceae}

Lemna minor L. (common duckweed); P; F; WAV; O-280; (APW:Araceae)

Spirodela polyrrhiza (L.) Schleid. (common duckmeat); P; F; WAV; O-279; (APW:Araceae)

Wolffia columbiana Karst. (Columbian watermeal); P; F; WAV; O-281; (APW:Araceae)

\section{Liliaceae}

Allium canadense L. (meadow garlic); P; F; DHV; O-124; (APW:Amaryllidaceae)

Camassia scilloides (Raf.) Cory (Atlantic camas); P; F; DHV; O-064; (APW:Asparagaceae)

Erythronium albidum Nutt. (white fawnlily); P; F; MF; O-007; (APW:Liliaceae)

*Liriope spicata (Thunb.) Lour. (creeping liriope); P; F; MF; O-204; (APW:Asparagaceae)

Nothoscordum bivalve (L.) Britton (crowpoison); P; F; DHV; O-026; (APW:Amaryllidaceae)

Polygonatum biflorum (Walter) Elliott (smooth Solomon's seal); P; F; MF; O-045; (APW:Asparagaceae)

\section{Poaceae}

Andropogon gerardii Vitman (big bluestem); P; G; DHV; O-227

Arundinaria gigantea (Walter) Muhl. (giant cane); P; G; BF; O-302

Bothriochloa saccharoides (Sw.) Rydb. (silver bluestem); P; G; DHV; 0-371

*Bromus catharticus Vahl (rescuegrass); A; G; DHV; O-029

Bromus pubescens Muhl. ex Willd. (hairy woodland brome); P; G; MF, BF; 0-076

*Bromus racemosus L. (bald brome); A; G; DHV; 0-122

${ }^{\star}$ Bromus sterilis L. (poverty brome); A; G; DHV; O-081

Chasmanthium latifolium (Michx.) Yates (Indian woodoats); P; G; MF, BF; 0-207

Cinna arundinacea L. (sweet woodreed); P; G; BF; O-326

${ }^{\star}$ Cynodon dactylon (L.) Pers. (Bermudagrass); P; G; DHV; O-304

Diarrhena obovata (Gleason) Brandenburg (obovate beakgrain); P; G; MF; 0-215 
Dichanthelium malacophyllum (Nash) Gould (softleaf rosette grass); P; G; MF; 0-148

Digitaria ciliaris (Retz.) Koeler (southern crabgrass); A; G; DHV; O-268

${ }^{\star}$ Echinochloa colona (L.) Link (jungle rice); A; G; DHV; O-347

Echinochloa muricata (P. Beauv.) Fernald (rough barnyardgrass); A; G; DHV, WAV; 0-218

*Eleusine indica (L.) Gaertn. (Indian goosegrass); A; G; DHV; O-239

Elymus villosus Muhl. ex Willd. (hairy wildrye); P; G; MF; 0-144

Elymus virginicus L. (Virginia wildrye); P; G; MF, BF; O-224

${ }^{\star}$ Eragrostis cilianensis (All.) Vign. ex Janchen (stinkgrass); A; G; DHV; O-376

Eragrostis hypnoides (Lam.) Britton, Sterns \& Poggenb. (teal lovegrass); A; G; WAV; 0-307

Eragrostis lugens Ness (morning lovegrass); P; G; DHV; O-370

${ }^{\star}$ Eragrostis pilosa (L.) P. Beauv. (Indian lovegrass); A; G; DHV; O-252

Festuca subverticillata (Pers.) Alexeev (nodding fescue); P; G; MF; 0-083

Hordeum pusillum Nutt. (little barley); A; G; DHV; O-028

†Muhlenbergia bushii Pohl (nodding muhly); P; G; BF; O-414

Panicum anceps Michx. (beaked panicgrass); P; G; MF, WAV; 0-206

Panicum dichotomiflorum Michx. (fall panicgrass); A; G; DHV; 0-356

Panicum rigidulum Bosc ex Nees (redtop panicgrass); P; G; WAV; 0-334

Panicum virgatum L. (switchgrass); P; G; DHV; O-226

*Paspalum dilatatum Poir. (dallisgrass); P; G; DHV; O-157

Paspalum floridanum Michx. (Florida paspalum); P; G; DHV; O-354

*Poa annua L. (annual bluegrass); A; G; DHV; O-021

${ }^{*}$ Poa pratensis L. (Kentucky bluegrass); P; G; DHV; O-198

Poa sylvestris A. Gray (woodland bluegrass); P; G; MF; 0-050

*Schedonorus pratensis (Huds.) P. Beauv. (meadow fescue); P; G; DHV; 0-102

Setaria parviflora (Poir.) Kerguélen (marsh bristlegrass); P; G; DHV; O-221

*Setaria pumila (Poir.) Roem. \& Schult. (yellow foxtail); A; G; DHV; 0-372

Sorghastrum nutans (L.) Nash (Indiangrass); P; G; DHV; O-323

*Sorghum halepense (L.) Pers. (Johnsongrass); P; G; DHV; O-208

Tridens flavus (L.) Hitchc. (purpletop tridens); P; G; DHV; O-270

Tridens strictus (Nutt.) Nash (longspike tridens); P; G; DHV; O-420

Tripsacum dactyloides (L.) L. (eastern gamagrass); P; G; DHV; O-137

\section{Smilacaceae}

Smilax bona-nox L. (saw greenbrier); P; V; MF, BF; 0-091

†Smilax lasioneura Hook. (Blue Ridge carrionflower); P; V; MF; 0-093

Smilax rotundifolia L. (roundleaf greenbrier); $\mathrm{P} ; \mathrm{V} ; \mathrm{BF} ; \mathrm{O}-185$

Smilax tamnoides L. (bristly greenbrier); P; V; MF, BF; 0-056

\section{Typhaceae}

*Typha angustifolia L. (narrowleaf cattail); P; F; WAV; 0-156 